University of Nebraska - Lincoln

DigitalCommons@University of Nebraska - Lincoln

2013

On the treatment of LUMO energies for their use as descriptors

Paul Burrow

Alberto Modelli

Follow this and additional works at: https://digitalcommons.unl.edu/physicsburrow

Part of the Atomic, Molecular and Optical Physics Commons

This Article is brought to you for free and open access by the Research Papers in Physics and Astronomy at DigitalCommons@University of Nebraska - Lincoln. It has been accepted for inclusion in Paul Burrow Publications by an authorized administrator of DigitalCommons@University of Nebraska - Lincoln. 


\title{
On the treatment of LUMO energies for their use as descriptors
}

\author{
P.D. Burrow ${ }^{1}$ and A. Modelli ${ }^{2,3}$ \\ 1 Department of Physics and Astronomy, University of Nebraska-Lincoln, Lincoln, USA \\ 2 Università di Bologna, Dipartimento di Chimica "G. Ciamician", Bologna, Italy \\ 3 Centro Interdipartimentale di Ricerca in Scienze Ambientali, Ravenna, Italy \\ Corresponding author — P. D. Burrow, email pburrow@unl.edu
}

\begin{abstract}
Calculated energies of lowest unoccupied molecular orbitals (LUMOs) are frequently employed as descriptors in studies of quantitative structure-activity relationships and linear free energy relationships involving electron transfer. However, the quantum chemical programs with which these are carried out, whether Hartree-Fock or density functional theory, do not treat orbitals of different character, for example, $\mathrm{C}=\mathrm{C}$ $\pi^{*}$ and $\mathrm{C}-\mathrm{Cl} \sigma^{*}$, consistently, nor is there consistency among different families of compounds. These problems can be ameliorated with the use of the experimental equivalent of the LUMO energy, the vertical attachment energy (VAE), or by shifting and scaling LUMO energies to a training set of available VAEs in similar compounds. Examples from the literature are used to illustrate these points.
\end{abstract}

Keywords: QSAR, LUMOs, vertical attachment energies, empirical energy scaling, reductive dehalogenation

\section{Introduction}

The energies of normally unoccupied molecular orbitals can be computed with relative ease using current quantum chemical programs. In particular, the lowest unoccupied molecular orbital (LUMO) plays a special role in reductive processes in which an electron from a molecular

Published in SAR and QSAR in Environmental Research, 2013 Vol. 24, No. 8, 647-659, http://dx.doi.org/10.1080/1062936X.2013.792873

Copyright (C) 2013 Taylor \& Francis. Used by permission.

Submitted 3 July 2012; revised 2 February 2013. 
donor anion or metallic surface is assumed to transfer into this orbital on an acceptor molecule. Calculated LUMO energies have therefore become a popular descriptor in studies of quantitative structure-activity relationships (QSARs) applied to reduction reactions in molecular families. LUMO energies are functions of the quantum chemical method employed. The LUMO of ethene determined by DFT/B3LYP/6-31G(d), for example, differs significantly from that given by HF/6-31G(d). However, the relative LUMO energies in a series of congeners are in broad agreement, and only these are usually considered to be of significance in QSAR studies.

According to Koopmans' Theorem [1], the normally empty orbital energies, that is, the virtual orbital energies (VOEs) of neutral molecules, are associated with the energies of the anion states that arise by addition of an electron to the neutral molecule and occupation of a formerly empty orbital. Unfortunately, it is not widely appreciated that many such anion states can be observed as sharp resonance structures appearing in the cross-sections for free electron scattering from the neutral molecules in the gas phase. Because electron attachment occurs on a time scale very much shorter than that for nuclear motion, the energies of these short-lived anion states are characteristic of the equilibrium geometry of the neutral molecule, as are LUMO energies, and are labelled vertical attachment energies (VAEs). If VAEs are available for the compounds of interest in a QSAR study, using these in place of calculated LUMO energies may provide more insight since they yield directly the energies of the reduced molecules in their neutral state geometries in the gas phase.

A more likely situation is that VAEs may be available only for a subset of a given molecular family of interest. In this case it is still possible to put calculated LUMO energies for a series of related molecules on an absolute energy scale relative to that of the neutral molecules by empirically shifting and scaling the VOEs to match the several experimentally determined VAEs that are available. These scaled virtual orbital energies (SVOEs) can then be used in place of the unknown VAEs. Because VAEs can only be measured for anion states whose energies are above those of the neutral molecules, that is, in the positive energy electron scattering continuum, negative values of VAE, corresponding to stable anion states, are not experimentally accessible. However, if a scaling can be determined from other family members with positive VAEs, SVOEs associated with these stable anion states can also be computed. 
The purpose of this contribution is not to promote LUMO energies as QSAR descriptors, but rather to focus on inconsistencies that arise from their use and to illustrate how in some cases it may be possible to derive additional information about the reaction properties. Our approach is straightforward. We consider several literature studies in which dehalogenation rates have been plotted against calculated LUMO energies, replace the latter with VAEs or SVOEs, and observe the ways in which the correlations, if any, are altered. We want to stress that it is not our purpose to further justify the use of such gas phase descriptors in reactions taking place in solution, or to examine whether LUMO energies are in fact the appropriate descriptors for a particular reaction mechanism. Our objective is solely to bring to the reader's attention problems that arise with calculated LUMO energies in differing molecular families. We begin with a short discussion of VAEs and VOE scaling.

\section{VAEs and SVOEs}

The anion states associated with the short-lived occupation of normally unoccupied molecular orbitals have been extensively explored using electron transmission spectroscopy (ETS) [2]. A brief discussion of the important concepts and the application to temporary anion states of hydrocarbons may be found elsewhere [3]. Although virtual orbital properties were employed early on as an aid in assigning the symmetries of the observed temporary anion states, the first extensive comparison of VAEs and VOEs was carried out by Heinrich et al. [4] using Hartree-Fock (HF) calculations with several choices of basis sets and the VAEs available at that time. For our purposes this paper is significant because it showed clearly that the LUMOs of chloro-alkanes, where $\sigma^{*}(\mathrm{C}-\mathrm{Cl})$ orbitals are involved, do not have the same correlation with VAE as that found in the LUMOs of substituted ethenes, which are $\pi^{*}(C=C)$ in character. A second observation of interest pointed out that use of basis sets with increasing amounts of diffuseness resulted in poorer agreement with experiment.

Although regression equations between VAE and VOE were not presented by Heinrich et al. [4], these issues have been explored in more detail by others $([5,6]$ and entries in the Appendix). The current status of VOE scaling can be summarized as follows: (a) because the temporary anion states associated with empty $\pi^{*}$ orbitals of unsaturated 
hydrocarbons are the sharpest and most prominent in the electron scattering spectra, these states have received the most attention, and the VAEs are the most accurately known; (b) the best fits to plots of VAE vs. VOE are obtained within given families of compounds, i.e. substituted ethenes or ethynes or aromatic ring compounds. Regressions to multiple family groups are still very useful but will display more scatter; (c) VAEs associated with empty $\sigma^{*}$ orbitals have been observed only for molecular bonds involving third-row or heavier atoms such as chlorine and bromine. These temporary anion states are broader in energy because of their short lifetimes and their repulsive potential surfaces as a function of the $\mathrm{C}-\mathrm{X}$ bond length. Consequently they are more difficult to observe and locate accurately. In unsaturated compounds, their overlap with $\pi^{*}$ anion states may also cause difficulties. The scaling equations for $\sigma^{*}(\mathrm{C}-\mathrm{Cl})$ and $\sigma^{*}(\mathrm{C}-\mathrm{Br})$ anion states are significantly different not only from those associated with $\pi^{*}$ anion states, but also from $\sigma^{*}$ anion states of different families of compounds, such as group 14 dimers [7]. This is therefore a source of inconsistency in unscaled LUMOs as we discuss below; and (d) although VOEs determined from any basis set can be used in principle to generate scaling equations, experience has shown that relatively small basis sets such as 6-31G(d), whether used with HF or density functional theory (DFT) calculations, provide the best results $[6,8]$.

\section{Rate constants and VAEs}

In this section we review an example in which measured VAEs were used to replace calculated LUMO energies. Scherer et al. [9], in a study of kinetic data [10] for dehalogenation of chlorinated alkanes and alkenes by zero-valent iron $\left(\mathrm{Fe}^{\circ}\right)$, found that calculated LUMO energies were the most successful in explaining the variation in surface area-normalized rate constants $(\mathrm{k})$. A plot of their result is shown in Figure 1, with values and molecular identifications given in Table 1. For purposes of comparison, we have assumed that the result for TCM (trichloromethane) is an outlier. The linear regression shown with the remaining compounds yields $r^{2}=0.93$.

The experimental VAEs for all the compounds are known [11-13] except for PCM (tetrachloromethane) and HCA (hexachloroethane). A close approximation to the VAEs of the latter two may be obtained 


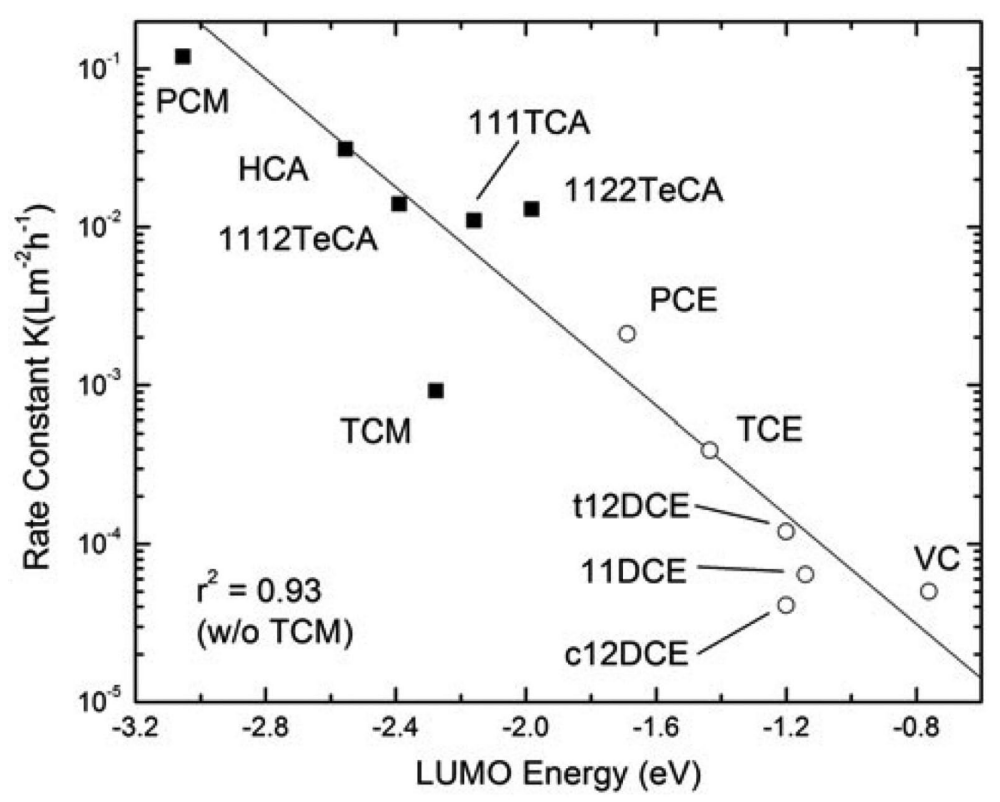

Figure 1. Surface-area normalized dechlorination rate constants $\mathrm{k}$ as a function of calculated LUMO energies of Scherer et al. [9]. The open circles indicate the ethenes. Excluding the point for TCM, the regression line yields $r^{2}=0.93$.

Table 1. Rate Constants, LUMO Energies and VAEs.

\begin{tabular}{|c|c|c|c|c|c|}
\hline Compound & Abbrev & $\mathrm{k}\left(\mathrm{Lm}^{-2} \mathrm{~h}^{-1}\right)^{\mathrm{a}}$ & $\begin{array}{l}\text { LUMO } \\
(\mathrm{eV})^{\mathrm{b}}\end{array}$ & $\begin{array}{l}\text { VAE } \\
(\text { LUMO) } \\
(\mathrm{eV})^{\mathrm{c}}\end{array}$ & $\begin{array}{l}\text { VAE } \\
(\mathrm{LUMO+1}) \\
(\mathrm{eV})^{\mathrm{c}}\end{array}$ \\
\hline Tetrachloromethane & PCM & $1.2 \times 10^{-1}$ & -3.054 & -0.24 (est) $\sigma^{*}$ & \\
\hline Trichloromethane & TCM & $9.2 \times 10^{-4}$ & -2.277 & $0.35 \sigma^{*}$ & \\
\hline Hexachloroethane & HCA & $3.1 \times 10^{-2}$ & -2.555 & 0.14 (est) $\sigma^{*}$ & \\
\hline 1,1,1,2_Tetrachloroethane & 1112TeCA & $1.4 \times 10^{-2}$ & -2.390 & $0.63 \sigma^{*}$ & \\
\hline 1,1,2,2_Tetrachloroethane & $1122 \mathrm{TeCA}$ & $1.3 \times 10^{-2}$ & -1.982 & $0.51 \sigma^{*}$ & \\
\hline 1,1,1_Trichloroethane & 111TCA & $1.1 \times 10^{-2}$ & -2.160 & $0.64 \sigma^{*}$ & \\
\hline Tetrachloroethene & PCE & $2.1 \times 10^{-3}$ & -1.689 & $0.3 \pi^{*}$ & $1.30 \sigma^{*}$ \\
\hline Trichloroethene & TCE & $3.9 \times 10^{-4}$ & -1.435 & $0.59 \pi^{*}$ & $1.59 \sigma^{*}$ \\
\hline cis_1,2_Dichloroethene & c12DCE & $4.1 \times 10^{-5}$ & -1.200 & $1.11 \pi^{*}$ & $2.30 \sigma^{*}$ \\
\hline trans_1,2_Dichloroethene & t12DCE & $1.2 \times 10^{-4}$ & -1.200 & $0.80 \pi^{*}$ & $2.22 \sigma^{*}$ \\
\hline 1,1_Dichloroethene & 11DCE & $6.4 \times 10-5$ & -1.140 & $0.76 \pi^{*}$ & $1.63 \sigma^{*}$ \\
\hline Vinyl chloride & VC & $5.0 \times 10^{-5}$ & -0.761 & $1.28 \pi^{*}$ & $2.84 \sigma^{*}$ \\
\hline
\end{tabular}

a. Refs. $[9,10]$.

b. Ref. [9].

c. Refs. [11-13]. 
from a scaling of empty $\sigma^{*}(\mathrm{C}-\mathrm{Cl})$ orbital energies in other chlorinated alkanes by plotting known VAEs against the LUMO energies computed using $\mathrm{HF} / 6-31 \mathrm{G}(\mathrm{d})$ [13]. The regression in that study showed that VAE = (VOE - 2.83)/1.11, where energies are in $\mathrm{eV}$. Thus, for other compounds in this family with unknown VAEs, we write that the scaled VOE (SVOE) is given in $\mathrm{eV}$ by

$$
\text { SVOE }=(\operatorname{VOE}-2.83) / 1.11
$$

For PCM and HCA, the SVOEs approximating their VAEs are -0.24 and $0.135 \mathrm{eV}$ respectively.

In Figure 2 we plot on a semi-log scale the rate constants tabulated by Scherer et al. [9] against the lowest VAEs of the compounds and the SVOEs for PCM and HCA. As noted previously [14], the data now clearly fall into two groups, namely, the saturated compounds in which the LUMOs are associated with $\left.\sigma^{*}(\mathrm{C}-\mathrm{Cl})\right)$ orbitals, and the unsaturated compounds in which the LUMOs are $\pi^{*}(\mathrm{C}=\mathrm{C})$ orbitals. Thus, the computation of the unscaled LUMO energies of Figure 1 does not treat $\sigma^{*}(\mathrm{C}-\mathrm{Cl})$ and $\pi^{*}(\mathrm{C}=\mathrm{C})$ orbitals consistently. This was evident earlier in

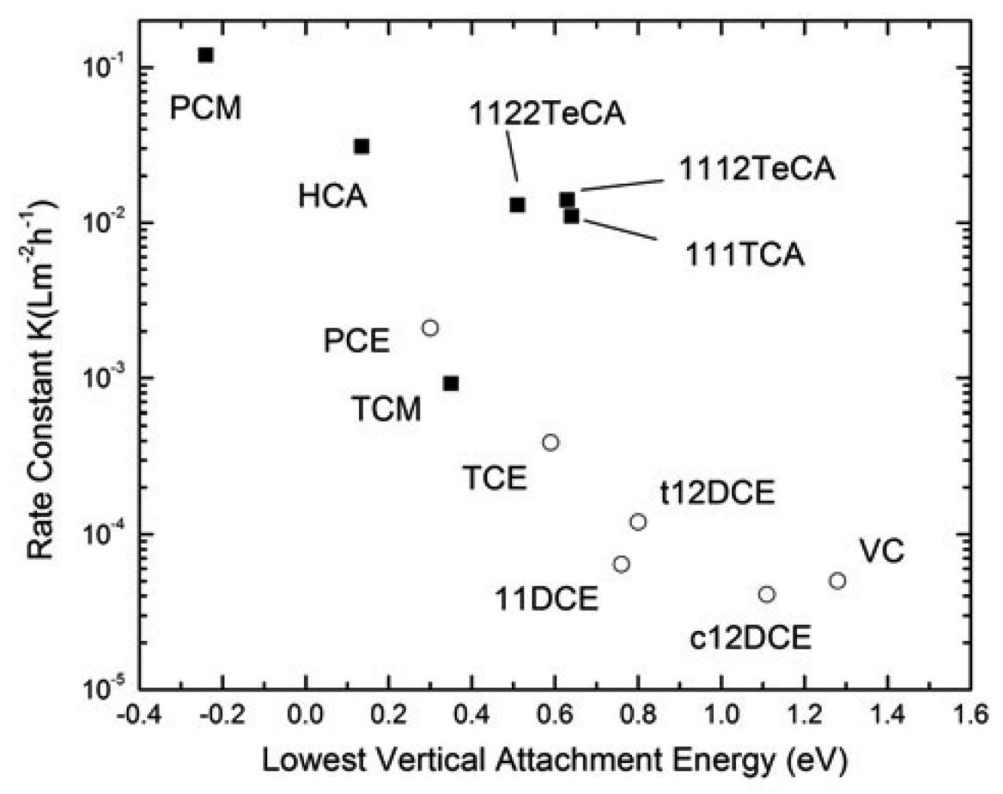

Figure 2. The rate constants of Figure 1 as a function of the measured VAEs corresponding to the LUMOs of the compounds. In the ethenes (open circles), these are $\mathrm{C}=\mathrm{C}$ $\pi^{*}$ in character, and in the remainder of the compounds, C-Cl $\sigma^{*}$. The values for PCM and HCA are SVOE estimates, see text. 
the comparisons of Heinrich et al. [4]. The reasons for this difference are complex and will not be dealt with here. Tratnyek et al. [15] in a later review have noted that the data in Figure 2, as published in Burrow et al. [14], led to a poorer $r^{2}$ than in Figure 1. However, Figure 2 shows that fitting the subgroups separately will provide stronger correlations.

We now extend this discussion beyond that of Burrow et al. [14]. Since it is not obvious a priori that electron transfer in the solution phase must take place initially into the $\pi^{*}$ orbital of the chloro-alkenes, it is useful to explore another possibility, namely that it is injection into the lowest orbital of $\sigma^{*}(\mathrm{C}-\mathrm{Cl})$ character in each of the compounds that is relevant. In the alkenes, this would correspond to electron transfer to the second lowest empty orbital (LUMO+1). The VAEs for these anion states are also available [11-13]. A plot of the rate constants as a function of these VAEs is shown in Figure 3. The regression line has $r^{2}=0.92$, with the omission of TCM as before, a reasonably strong correlation. It is not our purpose to pursue the implications about mechanism that Figures 2 and 3 suggest, but it appears that there are two possible conclusions. If electron transfer to the $\pi^{*}$ orbitals of the ethenes is indeed the initial step in these compounds, then for a given value of VAE, the rates in the

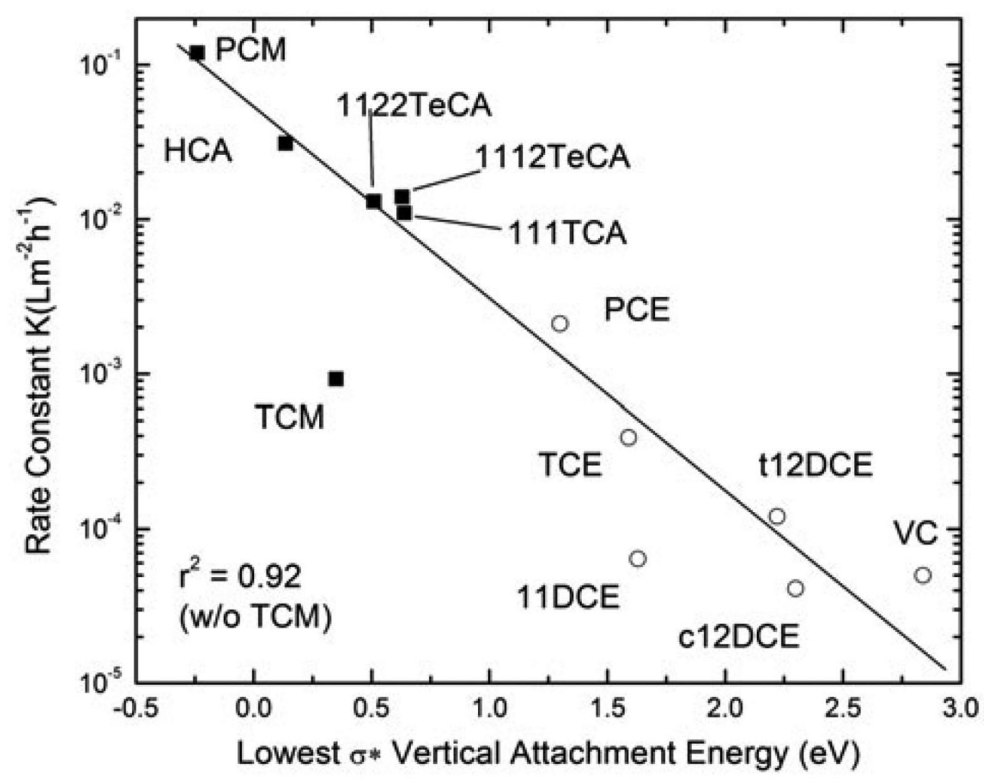

Figure 3. The rate constants of Figure 1 as a function of measured VAEs corresponding to attachment into the lowest orbital of $\mathrm{C}-\mathrm{Cl} \sigma^{*}$ character in each compound. In the ethenes, this corresponds to the LUMO+1. 
ethenes are substantially smaller than in the saturated compounds. On the other hand, Figure 3 suggests that if electron transfer occurs to the lowest $\sigma^{*}(\mathrm{C}-\mathrm{Cl})$ orbital, then both sets of compounds behave in a consistent manner as a function of attachment energy, but it is the LUMO+1 rather than the LUMO that plays the key role in the ethenes.

\section{Rate constants and SVOEs}

\subsection{Example A}

Next we consider an extensive analysis by Perlinger et al. [16] of linear free energy relationships for transformation of polyhalogenated methanes and ethanes in aqueous systems containing bulk reductants and electron transfer mediators. In particular we examine the results derived with iron porphyrin mediators. Our focus here is on their LUMO calculations which were carried out using DFT with the B3LYP functional and an extremely diffuse basis set, namely 6-311++g(d,p). In Figure 4 the

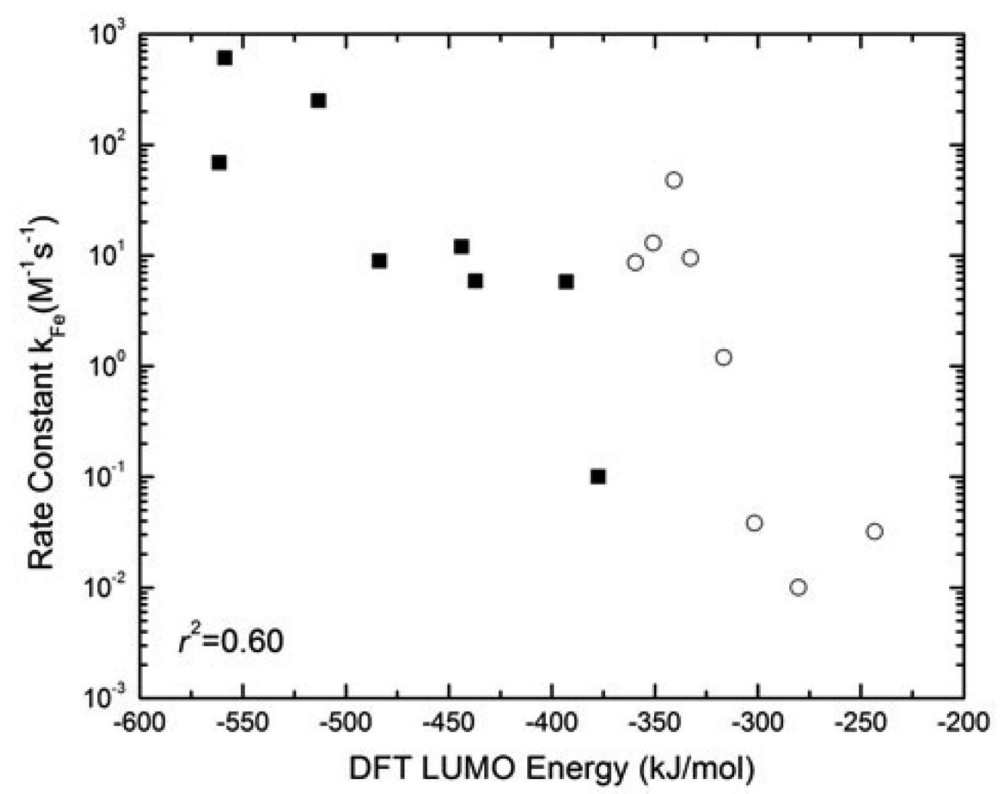

Figure 4. Rate constants of Perlinger et al. [16] plotted against LUMO energies calculated using DFT/ B3LYP/6-311++g(d,p) for polyhalogenated methanes (solid squares) and ethanes (open circles). The compounds are labelled in accordance with Table 2. An overall regression shows $r^{2}=0.60$. The regression through the ethanes alone yields $r^{2}=0.75$. 
measured rate constants $\mathrm{k}_{\mathrm{Fe}}$ are plotted as a function of these LUMO energies. For convenience the $\mathrm{C}_{1}$ and $\mathrm{C}_{2}$ compounds are distinguished, the solid squares representing the polyhalogenated methanes and the open circles the ethane-based compounds. Table 2 contains a listing of the molecules and the parameters of interest for this discussion. The compounds are numbered as indicated in Table 2. A regression through the entire data set has $r^{2}=0.60$, indicating considerable scatter. The regression through the $\mathrm{C}_{2}$ compounds alone yields $r^{2}=0.75$. We note in passing that the DFT LUMO energies of Perlinger et al. [16] are very close to a factor of two larger in magnitude than test calculations we carried out using the B3LYP/6-311++g(d,p) method.

Except for $\mathrm{CCl}_{4}$ and $\mathrm{CFCl}_{3}$, the substituted methane compounds all possess $\sigma^{*}$ LUMOs based on the C-Br bond. In the ethanes, the LUMOs are those associated with the $\mathrm{C}$ - $\mathrm{Cl}$ bond. Because VAEs for most of the compounds were not available, we utilize scaled VOEs for this study. The VOEs for all the compounds are computed with HF/6-31G(d), with the same basis set used for geometry optimization. For the $\sigma^{*}(\mathrm{C}-\mathrm{Cl}) \mathrm{LUMOs}$

Table 2. Rate constants, LUMO energies, C-X bond energies and scaled VOEs.

\begin{tabular}{lclccc} 
Compound & $\mathrm{k}_{\mathrm{Fe}}\left(\mathrm{M}^{-1} \mathrm{~s}^{-1}\right)^{\mathrm{a}}$ & $\begin{array}{l}\text { DFT LUMO } \\
\mathrm{kJ} / \mathrm{Mol}(\mathrm{eV})\end{array}$ & $\begin{array}{l}\text { DFT C-X Bond } \\
\text { Energy }\end{array}$ & $\begin{array}{l}\mathrm{HF} \text { VOE } \\
(\mathrm{eV})\end{array}$ & $\begin{array}{l}\text { SVOE } \\
(\mathrm{eV})\end{array}$ \\
\hline (1) $\mathrm{CHBrCl}_{2}$ & 5.8 & $-393(-4.073)$ & 262.1 & 2.523 & $-0.715^{\mathrm{b}}$ \\
(2) $\mathrm{CHBr}_{3}$ & 8.9 & $-483.6(-5.012)$ & 260.5 & 1.796 & $-1.449^{\mathrm{b}}$ \\
(3) $\mathrm{CHBr}_{2} \mathrm{Cl}$ & 12 & $-443.8(-4.600)$ & 261.3 & 2.117 & $-1.124^{\mathrm{b}}$ \\
(4) $\mathrm{CFBr}_{3}$ & 69 & $-561.4(-5.818)$ & 253.5 & 1.450 & $-1.798^{\mathrm{b}}$ \\
(5) $\mathrm{CBrCl}_{3}$ & 250 & $-513.1(-5.318)$ & 240.1 & 1.886 & $-1.358^{\mathrm{b}}$ \\
(6) $\mathrm{CBr}_{2} \mathrm{Cl}_{2}$ & 610 & $-558.7(-5.790)$ & 238.2 & 1.527 & $-1.721^{\mathrm{b}}$ \\
(7) $\mathrm{CFCl}_{3}$ & 0.10 & $-377.4(-3.911)$ & 292.2 & 2.950 & $0.108^{\mathrm{c}}$ \\
(8) $\mathrm{CCl}_{4}$ & 5.9 & $-437.1(-4.530)$ & 263.4 & 2.558 & $-0.245^{\mathrm{c}}$ \\
(9) $\mathrm{CH}_{3}-\mathrm{CCl}_{3}$ & 0.032 & $-243.3(-2.522)$ & 288.2 & 3.448 & $0.556^{\mathrm{c}}$ \\
(10) $\mathrm{CCl}_{3}-\mathrm{CCl}_{3}$ & 48 & $-340.6(-3.530)$ & 259.4 & 2.982 & $0.137^{\mathrm{c}}$ \\
(11) $\mathrm{CF}_{3}-\mathrm{CCl}_{3}$ & 8.6 & $-359.4(-3.725)$ & 272.2 & 3.102 & $0.245^{\mathrm{c}}$ \\
(12) $\mathrm{CHCl}_{2}-\mathrm{CCl}_{3}$ & 9.5 & $-332.7(-3.448)$ & 262.2 & 3.127 & $0.267^{\mathrm{c}}$ \\
(13) $\mathrm{CH}_{2} \mathrm{Cl}_{-}-\mathrm{CCl}_{3}$ & 1.2 & $-316.5(-3.280)$ & 270.5 & 3.233 & $0.363^{\mathrm{c}}$ \\
(14) $\mathrm{CF}_{2} \mathrm{Cl}_{-}-\mathrm{CFCl}_{2}$ & 0.038 & $-301.6(-3.126)$ & 295.1 & 3.548 & $0.647^{\mathrm{c}}$ \\
(15) $\mathrm{CHCl}_{2}-\mathrm{CHCl}_{2}$ & 0.010 & $-280.3(-2.905)$ & 294.2 & 3.649 & $0.738^{\mathrm{c}}$ \\
(16) $\mathrm{CF}_{2} \mathrm{Cl}_{-}-\mathrm{CCl}_{3}$ & 13 & $-350.7(-3.635)$ & 264.5 & 3.124 & $0.265^{\mathrm{c}}$ \\
\hline
\end{tabular}

a. Ref. [16].

b. C-Br $\sigma^{*}$ scaling.

c. C-Cl $\sigma^{*}$ scaling. 
we employ Equation (1) as given earlier. For the Br-bearing methanes, scaling is more problematic because of the smaller number of "training set" VAEs available and the small range spanned by their energies. For the present work, we use a scaling based on seven mono-Br alkanes and dibromomethane given by Pshenichnyuk et al. [17]:

$$
\text { SVOE }=(\text { VOE }-3.23) / 0.99
$$

Rate constants as a function of the scaled VOEs are shown in Figure 5. In contrast to the results of Figure 4, the halo-ethanes as a group now display a much tighter correlation, $r^{2}=0.97$, and it is clear that fitting the subgroup separately provides a statistically stronger correlation with a less ambiguous mechanistic interpretation. Some of the differences between Figures 4 and 5 could arise from the large basis set employed in the DFT calculations. A plot of the DFT VOEs of Perlinger et al. [16] against the unscaled VOEs computed with HF/6-31G(d), not shown, indicates that the compounds bearing one or more $\mathrm{H}$ atoms are treated differently, falling on opposite sides of the overall regression line from the rest, with few exceptions.

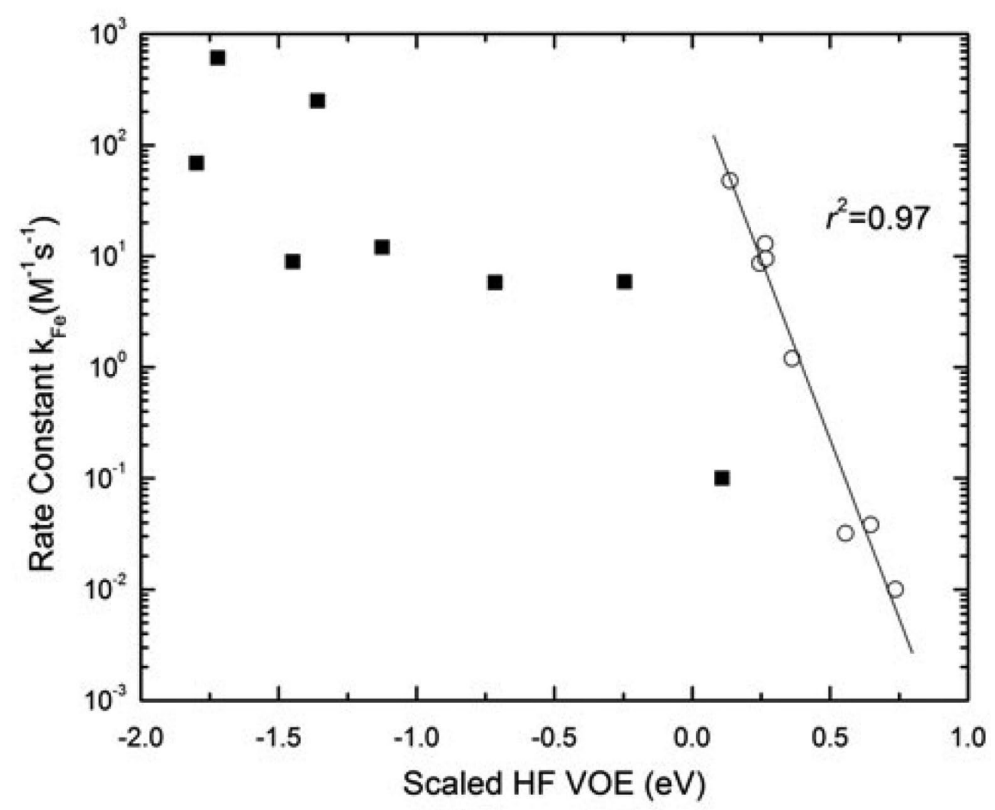

Figure 5. The rate constants of Perlinger et al. [16] plotted against scaled VOEs determined with HF/6-31G(d). Separate scalings are used for the C-Cl and C-Br $\sigma^{*}$ orbitals, see text. The ethane-based compounds (open circles) show a much higher correlation $\left(r^{2}=0.97\right)$. 
Perlinger et al. [16] note a rather poor correlation $\left(r^{2}=0.62\right)$ between the computed $\mathrm{C}-\mathrm{X}$ bond dissociation energies and their LUMO energies. When plotted against the HF scaled VOEs, not shown, the scatter among the $\mathrm{C}_{2}$ compounds is greatly reduced, quite similar to the contrast between Figures 4 and 5.

\subsection{Example B}

In a second example of the application of SVOEs, we examine a data set by Peijnenburg et al. [18] reporting reductive dehalogenation in anaerobic sediment-water systems in molecular families comprising halogenated benzenes, phenols and anilines. The data of Peijnenburg et al. [18] were previously discussed in terms of quantum chemically derived descriptors by Rorije et al. [19]. Table 3 gives a listing of the compounds and the log of the reductive rate coefficient $\mathrm{k}$. The three lowest VOEs computed with the HF/6-31G(d) basis set in the present work are shown, and in every case but one, $\mathrm{VOE}_{1}$ and $\mathrm{VOE}_{2}$ correspond to orbitals of $\pi^{*}$ character and $\mathrm{VOE}_{3}$ corresponds to an orbital of $\sigma^{*}$ character. The exception is $\mathrm{p} 4$ (2,4,6-tribromophenol) where, as indicated in the table, $\mathrm{VOE}_{2}$ is $\sigma^{*}(\mathrm{C}-\mathrm{Br})$ and $\mathrm{VOE}_{3}$ is $\pi^{*}$ in character. The scaled VOEs for $\sigma^{*}(\mathrm{C}-\mathrm{Cl})$ and $\sigma^{*}(\mathrm{C}-\mathrm{Br})$ used Equations (1) and (2) respectively, as given earlier. The $\pi^{*}$ VOEs are scaled using:

$$
\text { SVOE }=0.753(\text { VOE }-2.614)
$$

from No. 3 in the Appendix. The SVOE subscripts correspond to those of the VOEs and, because of the scaling, are not necessarily in order of increasing energy. In the rightmost column of Table 3, we tabulate the energy difference between the lowest orbital of $\sigma^{*}$ and $\pi^{*}$ character for discussion later. We note that although the experimental data [19] also included compounds containing iodine, the HF/6-31G(d) basis set does not permit our application. Since only two of the aniline compounds contained $\mathrm{Cl}$ or $\mathrm{Br}$ and this is insufficient to discern a trend, we have omitted them.

In contrast to the comparisons with unscaled LUMOs described earlier, for this data set we will employ our SVOEs to look for patterns in log $\mathrm{k}$ as a function of SVOE or combinations of SVOEs. In Figure 6 we plot $\log \mathrm{k}$ as a function of the first $\pi^{*}$ SVOE in each compound. The two lines 
Table 3. Log $\mathrm{k}, \mathrm{HF} / 6-31 \mathrm{G}(\mathrm{d})$ values for $\mathrm{VOE}_{1-3}{ }^{\mathrm{a}}$, scaled $\mathrm{VOEs}^{\mathrm{b}}$ and the scaled energy difference of the lowest orbitals of $\sigma^{*}$ and $\pi^{*}$ character. All orbital energies in $\mathrm{eV}$.

\begin{tabular}{|c|c|c|c|c|c|c|c|c|c|}
\hline Label & Compound & $\log k^{c}$ & $\mathrm{VOE}_{1}$ & $\mathrm{VOE}_{2}$ & $\mathrm{VOE}_{3}$ & $\mathrm{SVOE}_{1}$ & $\mathrm{SVOE}_{2}$ & $\mathrm{SVOE}_{3}$ & $\sigma^{*}-\pi^{*}$ \\
\hline b1 & 1,2-Dibromobenzene & -5.19 & 3.117 & 3.203 & 3.714 & 0.379 & 0.444 & 0.489 & 0.11 \\
\hline b2 & 1,2,4-Tribromobenzene & -4.69 & 2.685 & 2.794 & 3.228 & 0.054 & 0.136 & -0.002 & -0.053 \\
\hline b3 & 1-Bromo-2-chlorobenzene & -5.4 & 3.155 & 3.221 & 4.066 & 0.407 & 0.457 & 0.844 & 0.437 \\
\hline b4 & 1-Bromo-2,6-dichlorobenzene & -5.18 & 2.810 & 2.874 & 3.545 & 0.148 & 0.196 & 0.318 & 0.17 \\
\hline b5 & 1-Bromo-3,5-dichlorobenzene & -5.32 & 2.708 & 2.758 & 3.571 & 0.071 & 0.108 & 0.344 & 0.273 \\
\hline b6 & 1-Bromo-2-fluorobenzene & -5.21 & 3.187 & 3.686 & 4.458 & 0.431 & 0.807 & 1.240 & 0.809 \\
\hline b7 & 1-Bromo-2,4,5-trifluorobenzene & $e-4.39$ & 2.711 & 3.505 & 4.022 & 0.073 & 0.671 & 0.800 & 0.727 \\
\hline b8 & 1,2,3-Trichlorobenzene & -5.77 & 2.856 & 2.860 & 3.970 & 0.182 & 0.185 & 1.027 & 0.845 \\
\hline b9 & 1,2,4-Trichlorobenzene & -5.62 & 2.801 & 2.808 & 4.099 & 0.141 & 0.146 & 1.143 & 1.002 \\
\hline a1 & 4-Bromo-2,6-dichloroaniline & -4.26 & 2.858 & 3.398 & 3.738 & 0.184 & 0.59 & 0.513 & 0.329 \\
\hline a2 & 2,4,6-Trichloroaniline & -5.47 & 2.854 & 3.449 & 4.111 & 0.181 & 0.629 & 1.154 & 0.973 \\
\hline $\mathrm{p} 1$ & 2-Bromophenol & -4.29 & 3.422 & 4.033 & 4.398 & 0.608 & 1.069 & 1.180 & 0.572 \\
\hline $\mathrm{p} 2$ & 2,4-Dibromophenol & -4.03 & 2.987 & 3.537 & 3.679 & 0.281 & 0.695 & 0.454 & 0.173 \\
\hline p3 & 2,6-Dibromophenol & -4.18 & 2.985 & 3.639 & 3.719 & 0.279 & 0.772 & 0.494 & 0.215 \\
\hline $\mathrm{p} 4$ & 2,4,6-Tribromophenol & -3.88 & 2.595 & $3.069 \sigma^{*}$ & $3.192 \pi^{*}$ & 0.014 & -0.163 & 0.435 & -0.177 \\
\hline p5 & 2-Bromo-4-chlorophenol & -4.32 & 2.990 & 3.593 & 3.912 & 0.283 & 0.737 & 0.689 & 0.406 \\
\hline p6 & 2,3-Dichlorophenol & -4.83 & 3.097 & 3.692 & 4.446 & 0.364 & 0.812 & 1.456 & 1.092 \\
\hline p7 & 2,6-Dichlorophenol & -4.80 & 3.064 & 3.669 & 4.572 & 0.339 & 0.794 & 1.569 & 1.23 \\
\hline p8 & 2,4,6-Trichlorophenol & -4.64 & 2.656 & 3.258 & 3.954 & 0.0316 & 0.485 & 1.013 & 0.981 \\
\hline p9 & 2,3,5,6-Tetrachlorophenol & -4.59 & 2.406 & 3.002 & 3.572 & -0.157 & 0.292 & 0.668 & 0.825 \\
\hline p10 & 2,4-Dichloro-6-methylphenol & -5.02 & 3.068 & 3.727 & 4.628 & 0.342 & 0.838 & 1.620 & 1.278 \\
\hline
\end{tabular}

a. Present work.

b. $\pi^{*}$ scaling: SVOE $=0.753(\mathrm{VOE}-2.614), \sigma^{*}(\mathrm{C}-\mathrm{Cl})$ scaling: $\mathrm{SVOE}=(\mathrm{VOE}-2.83) / 1.11, \sigma^{*}(\mathrm{C}-\mathrm{Br})$ scaling: $\mathrm{SVOE}=(\mathrm{VOE}-3.23) / 0.99$. c. Ref. [19].

are drawn only to guide the eye to various groupings. The compounds along the lower line all contain three halogens except for b7 which has one $\mathrm{Br}$ and three $\mathrm{F}$ atoms. Along the upper line, the compounds contain two halogens. Of the extreme points $\mathrm{p} 9$ and $\mathrm{p} 1$, the former contains four halogens and the latter, only one. Thus, the grouping of these compounds along the horizontal axis reflects in part the inductive stabilization of the $\pi^{*}$ orbitals with increasing halogen substitution.

In Figure 7 we plot log $\mathrm{k}$ against both the lowest $\pi^{*}$ (filled squares) and $\sigma^{*}$ (open circles) SVOEs. Again, the lines are only to guide the eye. The $\sigma^{*}$ SVOEs also fall primarily into two groups but with rather more scatter than found in the $\pi^{*}$ SVOEs. The existence of multiple groups makes it clear that log $\mathrm{k}$ is not a function of SVOE alone, whether $\pi^{*}$ or $\sigma^{*}$. 


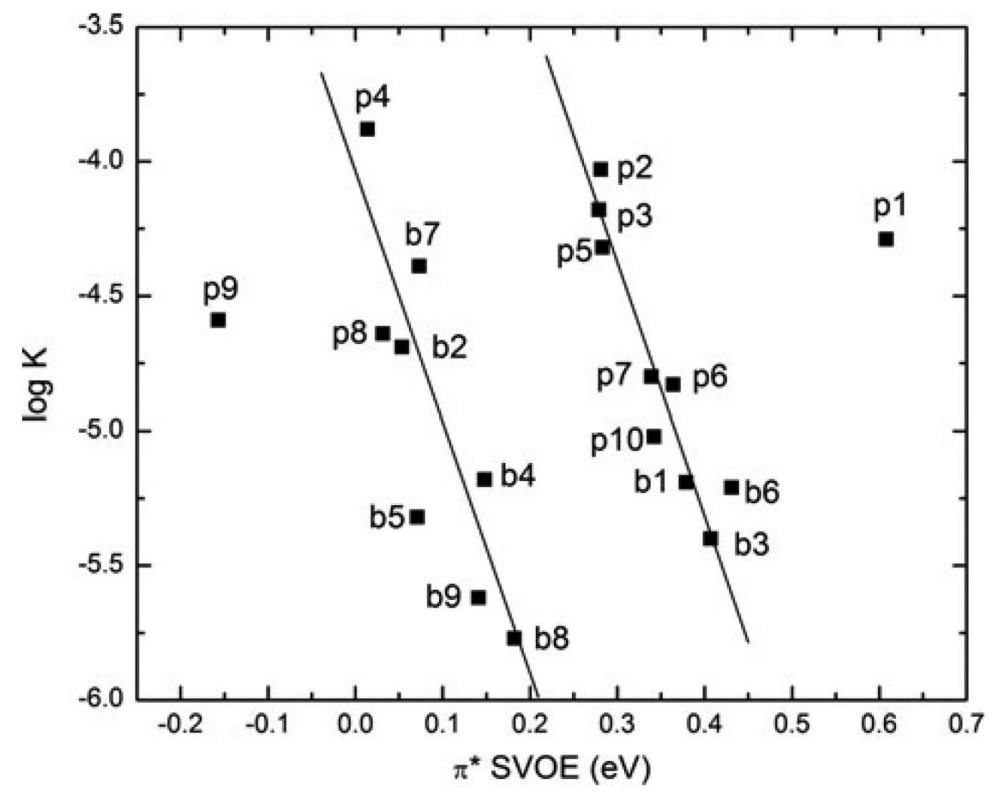

Figure 6. Log k from Rorije et al. [19] is plotted as a function of the lowest $\pi^{*}$ SVOE. The lines are only to guide the eye to the groupings.

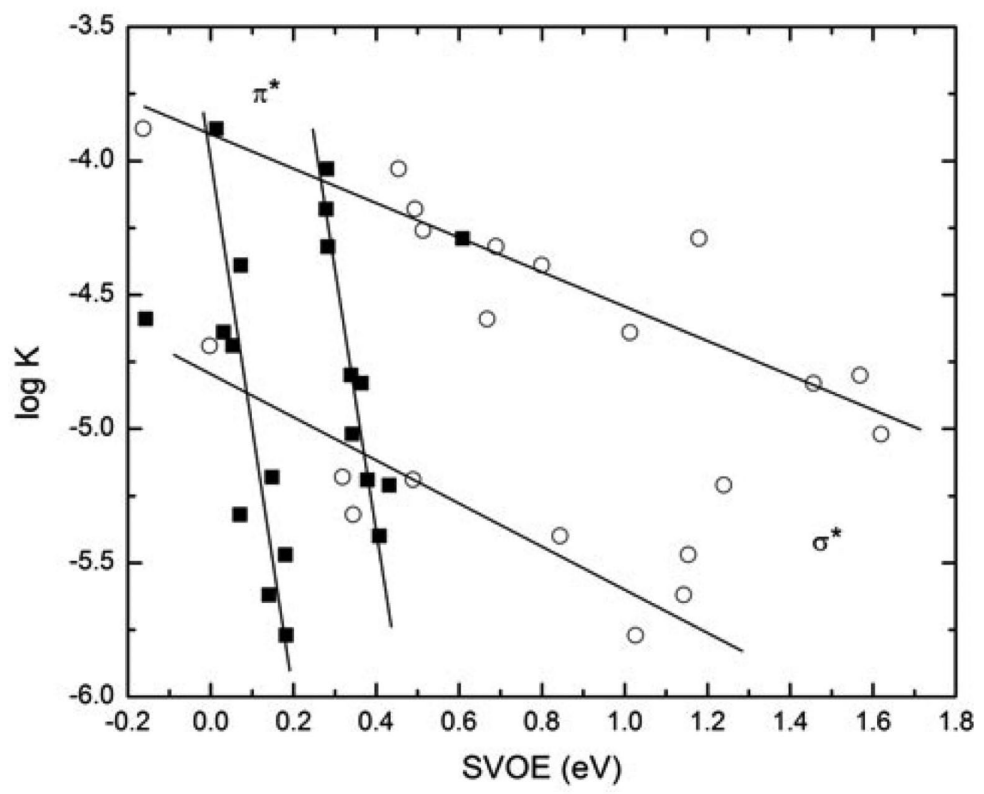

Figure 7. Log $\mathrm{k}$ is plotted as a function of the SVOEs of the lowest $\pi^{*}$ and $\sigma^{*}$ orbitals. The $\pi^{*}$ SVOEs are shown as filled squares and the $\sigma^{*}$ SVOEs as open circles. The lines are only to guide the eye. 


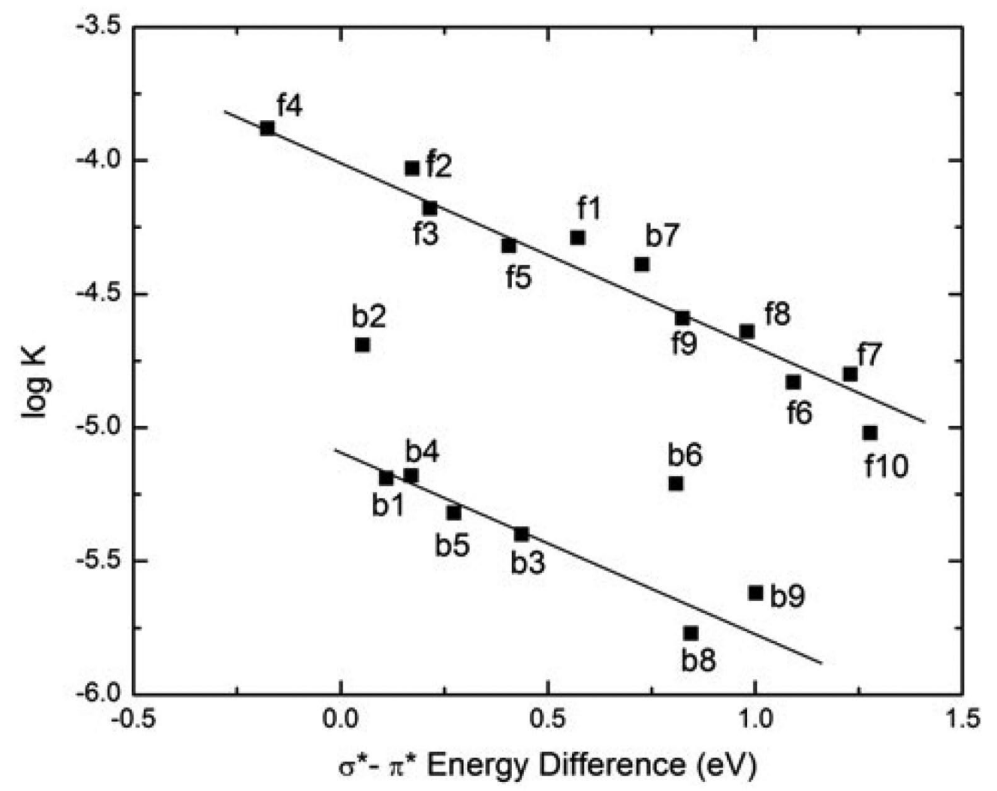

Figure 8. $\log \mathrm{k}$ is plotted as a function of the difference in energy of the lowest $\sigma^{*}$ and $\pi^{*}$ SVOEs in each compound.

The availability of the SVOEs makes it possible to search for other patterns in these data. For example, it may be of mechanistic interest to examine the extent to which the compounds with the highest rate coefficients tend to have $\pi^{*}$ and $\sigma^{*}$ SVOEs relatively close together. We explore this in Figure 8 by plotting log $\mathrm{k}$ against the $\sigma^{*}-\pi^{*}$ energy difference given in Table 3. The data fall largely into two groups, as suggested by the two lines, although b2 and b6 have intermediate values.

The dependence on the energy difference may be a consequence of the shapes of the anion curves. Because the $\sigma^{*}$ anion states are largely repulsive as a function of the $\mathrm{C}$ - $\mathrm{Br}$ or $\mathrm{C}$ - $\mathrm{Cl}$ bond stretch while the $\pi^{*}$ anions have a minimum along this coordinate similar to that of the neutral molecules, the location of the crossing point of the two curves will vary greatly with the vertical separation of the two anion states. When the spacing is small, the $\sigma^{*}$ curve will pass through the ground and lowest vibrational levels of the $\pi^{*}$ state, and the efficiency of transfer from nominally $\pi^{*}$ anions to $\sigma^{*}$ may be highest, with out-of-plane vibrational modes breaking the $\pi^{*} / \sigma^{*}$ symmetry and coupling the two anion states.

It should be pointed out that the complexity of the reaction medium, including the presence and growth of microorganisms, can create 
concern about the reliability of the data set of Peijnenburg et al. [18]. Consequently, caution is warranted in the interpretation of Figures 6-8. Nevertheless, the use of SVOEs for the lowest few anion states in each compound unveils the existence of correlations with the reported rate constants that would not be apparent with the simple use of LUMO energies alone, and may allow novel ways to explore the possible mechanisms for electron transfer.

\section{Conclusions}

Comparisons of calculated LUMO energies with experimentally determined VAEs have shown inconsistencies in the computed values among different families of compounds and among different substituents. It is important to note that LUMO energies themselves depend on the computational method and have no physical meaning. However, by shifting and scaling empty molecular orbital energies to match measured VAEs in other related molecules, these inconsistencies can be greatly reduced for this descriptor. In addition to the improvement of data correlation within a given molecular family as shown here with examples from the literature, the availability of VAEs and SVOEs may allow additional insight to electron transfer mechanisms. In particular, the importance of the role played by LUMO+1 in planar unsaturated halo-substituted compounds has been illustrated.

This paper has been directed primarily to those investigators who utilize the relatively simple HF or DFT calculations of virtual orbital energies for their comparisons with reductive rate coefficients. For completeness we should point out that attempts to calculate VAEs more accurately by quantum chemical means, as opposed to direct electron scattering methods, continue to develop. As in many areas of chemistry, DFT approaches are actively being explored. Entry to a portion of these much more complex calculations in the literature may be found, for example, in studies by Tozer and co-workers [20-22].

Acknowledgments AM thanks the Italian Ministero dell'Istruzione, dell'Università e della Ricerca for financial support (PRIN 2009SLKFEX). PDB wishes to thank Gordon Gallup for computation of some of the VOEs used in this work. 


\section{References}

[1] T. Koopmans, Über die Zuordnung von Wellenfunktionen und Eigenwerten zu den Einzelnen Elektronen Eines Atoms, Physica 1 (1934), pp. 104-113. Figure 8. Log $\mathrm{k}$ is plotted as a function of the difference in energy of the lowest $\sigma^{*}$ and $\pi^{*}$ SVOEs in each compound.

[2] L. Sanche and G.J. Schulz, Electron Transmission Spectroscopy: Rare Gases, Phys. Rev. A 5 (1972), pp. 1672-1683.

[3] K.D. Jordan and P.D. Burrow, Studies of the temporary anion states of unsaturated hydrocarbons by electron transmission spectroscopy, Acc. Chem. Res. 11 (1978), pp. 341-348.

[4] N. Heinrich, W. Koch, and G. Frenking, On the use of Koopmans' theorem to estimate negative electron affinities, Chem. Phys. Let. 124 (1986), pp. 20-25.

[5] D. Chen and G.A. Gallup, The relationship of the virtual orbitals of self-consistentfield theory to temporary negative ions in electron scattering from molecules, J. Chem. Phys. 93 (1990), pp. 8893-8901.

[6] S.W. Staley and J.T. Strnad, Calculation of the energies of $\pi^{*}$ negative ion resonance states by the use of Koopmans' Theorem, J. Phys. Chem. 98 (1994), pp. 116-121.

[7] A. Modelli and L. Szepes, Electron attachment to the mixed dimers (CH3)3M$M^{\prime}(\mathrm{CH} 3) 3$, with $M, M^{\prime}=S i, G e$, Sn, and correlation with the calculated $\sigma^{*}$ virtual orbital energies, Chem. Phys. 286 (2003), pp. 165-172.

[8] A. Modelli, Electron attachment and intramolecular electron transfer in unsaturated chloroderivatives, Phys. Chem. Chem. Phys. 5 (2003), pp. 2923-2930.

[9] M. Scherer, B.A. Balko, D.A. Gallagher, and P.G. Tratnyek, Correlation analysis of rate constants for dechlorination by zero-valent iron, Environ. Sci. Technol. 32 (1998), pp. 3026-3033.

[10] T.L. Johnson, M.M. Scherer, and P.G. Tratnyek, Kinetics of halogenated organic compound degradation by iron metal, Environ. Sci. Technol. 30 (1996), pp. 2634-2640.

[11] P.D. Burrow, A. Modelli, N.S. Chiu, and K.D. Jordan, Temporary $\Sigma$ and $\Pi$ anions of the chloroethylenes and chlorofluoroethylenes, Chem. Phys. Let. 82 (1981), pp. 270-276.

[12] P.D. Burrow, A. Modelli, N.-S. Chiu, and K.D. Jordan, Temporary negative ions in the chloromethanes, $\mathrm{CHCl} 2 \mathrm{~F}$ and $\mathrm{CCl} 2 \mathrm{~F} 2$; characterization of the $\sigma^{*}$ orbitals, J. Chem. Phys. 77 (1982), pp. 2699-2701.

[13] K. Aflatooni, G.A. Gallup, and P.D. Burrow, Temporary anion states of dichloroalkanes and selected polychloroalkanes, J. Phys. Chem. A 104 (2000), pp. 7359-7369.

[14] P.D. Burrow, K. Aflatooni, and G.A. Gallup, Dechlorination rate constants on iron and the correlation with electron attachment energies, Environ. Sci. Technol. 34 (2000), pp. 3368-3371.

[15] P.G. Tratnyek, E.J. Weber, and R.P. Schwarzenbach, Quantitative structure-activity relationships for chemical reductions of organic contaminants, Environ. Toxicol. Chem. 22 (2003), pp. 1733-1742. 
[16] J.A. Perlinger, R. Venkatapath, and J.F. Harrison, Linear free energy relationships for polyhalogenated alkane transformation by electron-transfer mediators in model aqueous systems, J. Phys. Chem. A 104 (2000), pp. 2752-2763.

[17] S.A. Pshenichnyuk, N.L. Asfandiarov, and P.D. Burrow, A relation between energies of the shortlived negative ion states and energies of unfilled molecular orbitals for a series of bromoalkanes, Russian Chem. Bull., Internat. Edit. 56 (2007), pp. 1268-1270.

[18] W.J.G.M. Peijnenburg, M.J. 't Hart, H.A. den Hollander, D. van de Meent, H.H. Verboom, and N. L. Wolfe, Reductive transformations of halogenated aromatic hydrocarbons in anaerobic water-sediment systems: Kinetics, mechanisms and products, Environ. Toxicol. Chem. 11 (1992), pp. 289-300.

[19] E. Rorije, J.H. Langenberg, J. Richter, and W.J.G.M. Peijnenburg, Modeling reductive dehalogenation with quantum chemically derived descriptors, SAR QSAR Environ. Res. 4 (1995), pp. 237-252.

[20] A.M. Teale, F. De Proft, and D.J. Tozer, Orbital energies and negative electron affinities from density functional theory: Insight from the integer discontinuity, J. Chem. Phys. 129 (2008), 044110.

[21] B. Hajgató, M.S. Deleuze, D.J. Tozer, and F. De Proft, $A$ benchmark theoretical study of the electron affinities of benzene and linear acenes, J. Chem. Phys. 129 (2008), 084308.

[22] A. Borgoo and D.J. Tozer, Negative electron affinities from DFT: Influence of asymptotic exchange-correlation potential and effective homogeneity under density scaling, J. Phys. Chem. A 116 (2012), pp. 5497-5500.

[23] M.J. Frisch, G.W. Trucks, H.B. Schlegel, G.E. Scuseria, M.A. Robb, J.R. Cheeseman, G. Scalmani, V. Barone, B. Mennucci, G.A. Petersson, H. Nakatsuji, M. Caricato, X. Li, H.P. Hratchian, A.F. Izmaylov, J. Bloino, G. Zheng, J.L. Sonnenberg, M. Hada, M. Ehara, K. Toyota, R. Fukuda, J. Hasegawa, M. Ishida, T. Nakajima, Y. Honda, O. Kitao, H. Nakai, T. Vreven, J.A. Montgomery Jr., J.E. Peralta, F. Ogliaro, M. Bearpark, J.J. Heyd, E. Brothers, K.N. Kudin, V.N. Staroverov, R. Kobayashi, J. Normand, K. Raghavachari, A. Rendell, J.C. Burant, S.S. Iyengar, J. Tomasi, M. Cossi, N. Rega, J.M. Millam, M. Klene, J.E. Knox, J.B. Cross, V. Bakken, C. Adamo, J. Jaramillo, R. Gomperts, R.E. Stratmann, O. Yazyev, A.J. Austin, R. Cammi, C. Pomelli, J.W. Ochterski, R.L. Martin, K. Morokuma, V.G. Zakrzewski, G.A. Voth, P. Salvador, J.J. Dannenberg, S. Dapprich, A. D. Daniels, O. Farkas, J.B. Foresman, J.V. Ortiz, J. Cioslowski, and D.J. Fox, Gaussian 09, Revision A.02, Gaussian, Inc., Wallingford CT, 2009.

[24] K. Aflatooni, G.A. Gallup, and P.D. Burrow, Electron attachment energies of the DNA bases, J. Phys. Chem. A 102 (1998), pp. 6205-6207.

[25] A. Scheer and P.D. Burrow, $\pi^{*}$ orbital system of alternating phenyl and ethynyl groups: Measurements and calculations, J. Phys. Chem. B 110 (2006), pp. 17751-17756.

[26] P.D. Burrow, G.A. Gallup, and A. Modelli, Are there $\pi^{*}$ shape resonances in electron scattering from phosphate groups? J. Phys. Chem. A 112 (2008), pp. 4106-4113. 


\section{Appendix}

For reference we list here a number of studies that have explored the scaling of VOEs and experimental measurements of VAEs. The compound families are given, along with the type of calculation, HF or DFT, the basis sets employed and the resulting scaling equations. All energies are expressed in $\mathrm{eV}$. In the present work, VOEs were calculated with the Gaussian suite of programs [23].

(1) From Chen and Gallup [5], obtained with HF/6-31G calculations for the empty $\pi^{*}$ VOEs of the compounds listed in Heinrich et al. [4], SVOE $=0.76(\mathrm{VOE}-2.33)$. The compounds consisted of substituted ethylenes, allene/acetylenes, carbonyls and substituted methanes.

(2) Staley and Strnad [6] list a great number of scalings with various basis sets. Their best results were found with HF/D95v//MP2/631G(d) for $\pi^{*}$ orbitals, yielding SVOE $=0.73865$ (VOE -1.90889$)$. The compounds comprised a broad range of mono-, di-, and tri-enes, both linear and cyclic, benzene and naphthalene.

(3) For application to the DNA bases [24], a $\pi^{*}$ orbital scaling based on the LUMOs of benzene, naphthalene, pyrimidine and pyridine, and computed with HF/6-31G(d), gives SVOE $=0.753(\mathrm{VOE}-2.614)$. (The earlier version in Aflatooni et al. [24] used geometry optimization at 3-21G(d) rather than 6-31G(d)).

(4) Modelli [8] has derived a scaling for $52 \pi^{*}$ orbitals using DFT with B3LYP/6-31G(d) and found SVOE $=0.8054$ (VOE + 1.5035) for alkenes, carbonyl, benzenoid and heterocyclic hydrocarbons. This reference also discusses the deleterious effects of using more diffuse basis sets.

(5) Scheer and Burrow [25] present results in acetylene, benzene and several molecules containing alternating phenyl and ethynyl groups for all the $\pi^{*} \mathrm{MOs}$, not just the LUMOs. The HF/ 6-31G(d) scaling is given by SVOE $=0.592(\mathrm{VOE}-2.22)$. The DFT scaling, B3LYP $/ 6-31 \mathrm{G}(\mathrm{d})$ yields SVOE $=0.806(\mathrm{VOE}+1.14)$.

(6) Modelli and Szepes [7] derived several linear regressions found with HF and B3LYP calculations for the $\sigma^{*} \mathrm{MOs}$ of $(\mathrm{CH} 3) 3 \mathrm{M}-\mathrm{M}^{\prime}\left(\mathrm{CH}_{3}\right)_{3}$ group 14 dimers, where $\mathrm{M}$ and $\mathrm{M}^{\prime}=\mathrm{Si}, \mathrm{Ge}, \mathrm{Sn}: \mathrm{HF} / 6-31 \mathrm{G}(\mathrm{d}): \mathrm{SVOE}=1.521$ (VOE - 3.177); HF/LanL2DZ: SVOE = 0.623(VOE - 0.363); B3LYP/6-31G(d): SVOE = 1.799(VOE - 0.046); B3LYP/LanL2DZ: SVOE $=0.779(\mathrm{VOE}+1.941)$.

(7) Burrow et al. [26] find a scaling obtained with the $\sigma^{*}(\mathrm{C}-\mathrm{Cl})$ VAEs of 13 chloroalkanes and the corresponding B3LYP/6-31G(d) VOEs: SVOE $=0.8111(\mathrm{VOE}+$ 1.9846). 